\title{
Elasto-plastic Finite Element Analysis of an Earth-Rockfill Dam Based on Generalized plasticity model
}

\author{
Jin congcong ${ }^{1,2, a}$, Chi shichun ${ }^{1,2}$ \\ ${ }^{1}$ State Key Laboratory of Coastal and Offshore Engineering, Dalian University of Technology, Dalian, \\ China \\ ${ }^{2}$ Institute of Earthquake Engineering, Faculty of Infrastructure Engineering, Dalian University of \\ Technology, Dalian, China \\ ajincong3623@mail.dlut.edu.cn
}

\begin{abstract}
Keywords: earth-rockfill dam; PZC elasto-plastic constitution model; DYNE3WAC program; impounding period.
\end{abstract}

Abstract. The Pastor-Zienkiewicz-Chan model based on the generalized plasticity theory can simulate the main materials of the earth-rockfill quite well. The three-dimensional modeling of a typical earth-rockfill dam was modeled by HYPERMESH and was implemented into the three-dimensional elasto-plastic program DYNE3WAC through the development of the interface based on FORTRAN. Therefore, the DYNE3WAC can analysized the typical earth-rockfill dam in the impoundingt period and reasonable analysis the dam displacement along the river and settlement and the pore water pressure which can satisfy the dam static development rules. The results show that displacement and pore water pressure are in accordance with the general rule of the earth-rockfill dam and can prepare for the next dynamic analysis.

\section{Introduction}

The earth-rockfill dam has the advantages of convenient selection, low requirement of foundation, convenient construction compared to other dam type. Therefore, this kind of dam has been in the priority position in the construction of dam construction in the world. As China's demand for clean energy continues to increase, the demand for the use of hydropower is also increasingly strong. So many earth-rockfill dams were proposed and builded [1]. Failures of the earth-rockfill dams can cause catastrophic economic damage and loss of lives. Numerical analysis based on constitutive model of geotechnical materials is one of the effective ways to conduct analyses and safety assessment of dam. Therefore, it is very vital to analyze the dam with the more advanced and reasonable numerical method for the earth-rock dam [2].

The numerical simulation of earth-rock dam usually includes static and dynamic parts, in which static calculation is able to analyze the stress and deformation of dam body during construction and impounding period. The numerical simulation of geotechnical engineering, especially the rapid development of finite element method, makes the numerical simulation level of earth-rock dam more and more high. Among them, the constitutive model of soil and rockfill material are the key to the accuracy and efficiency of calculation. Mroz and Zienkiewicz [3] proposed the generalized plasticity theory for the first time in 1984, and later developed through many scholars. The theoretical model has been applied to a certain degree in academia and civil engineering project[4-6].

\section{Pastor-Zienkiewicz-Chan MODEL}

The generalized plasticity theory does not need to establish explicit yield function and plastic potential function, which can describe the plastic deformation of soil during loading and unloading, and use non-associated flow law, which is suitable for describing the engineering mechanical properties of rock and soil. The generalized plasticity theory was proposed by Zienkiewicz and Mroz 
to model the behaviors of sand under monotonic and cyclic loading. The key futures of this theory are that neither yield surface nor plastic potential surface needs to be defined explicitly, and consistency law is not required to determine plastic modulus.

Later, Pastor, Zienkiewicz and Chan [7] based on this theory, proposed PZC elasto-plastic constitutive model.In the theory, the total strain increment is divided into elastic and plastic components [8]. The relationships between elastic volumetric and shear strain increments and stress increments are defined as

$d \varepsilon=d \varepsilon^{e}+d \varepsilon^{p}$

where $d \varepsilon^{e}$ and $d \varepsilon^{p}$ elastic and plastic strain increments, respectively.

The relationship between strain and stress increments is expressed as

$$
d \sigma=D^{e p} d \varepsilon
$$

where $D^{e p}$ is the elasto-plastic sti ness tensor given as

$$
D^{e p}=D^{e}-\frac{D^{e}: n_{g L / U}: \mathrm{n}^{T}: D^{e}}{H_{L / U}+\mathrm{n}^{T}: D^{e}: n_{g L / U}}
$$

where $n, n_{g L / U}$ and $H_{L / U}$ represent loading direction vector, plastic flow direction vector under loading and unloading condition, and plastic modulus for loading and unloading, respectively.

The elastic shear and bulk moduli $G$ and $K$ depend both on the sand density and on mean effective stress. They are given by the following relations:

$G=G_{0}\left(p^{\prime} / p_{a}\right)$

$K=K_{0}\left(p^{\prime} / p_{a}\right)$

where $G_{0}$ and $K_{0}$ represent shear and bulk modulus number and $p_{a}$ atmospheric pressure that is equal to $101.325 \mathrm{kPa}$.

The stress-dilatancy relationship is provided by Frossard [9] :

$d_{g}=\frac{d \varepsilon_{v}^{p}}{d \varepsilon_{s}^{p}}=\left(1+\alpha_{g}\right)\left(M_{g}-\eta\right)$

where $d \varepsilon_{v}^{p}$ and $d \varepsilon_{s}^{p}$ represent incremental plastic volumetric and plastic deviatoric strains, respectively. $\alpha_{g}$ is a model parameter and $M_{g}$ is the slope of the critical state line on p-q plane, $\eta(=q / p)$ is the stress ratio.

Accordingly, dilatancy will equal zero when the stress ratio equals $M_{g}$. Therefore, the $M_{g}$ is dependent on the angle of internal friction at the critical state $\phi_{c}$, and Lode's angle, $\theta$ :

$$
\begin{aligned}
& M_{g}=\frac{6 \sin \phi_{c}}{3-\sin \phi_{c} \sin 3 \theta} \\
& \sin 3 \theta=\frac{3 \sqrt{3}}{2} \frac{J_{3}}{J_{2}^{3 / 2}}
\end{aligned}
$$

In PZC model, yield and potential surfaces are not defined explicitly. Instead, gradient vectors of these surfaces are used. Gradient vector of yield surface is known as loading direction vector $n$ and gradient vector of potential surface as plastic flow direction vector $n_{g}$. The flow rule is assumed to be non-associated so the mentioned two vectors are not same and $n_{g L}$ is expressed as

$n_{g L}=\frac{1}{\sqrt{1+d_{g}^{2}}}\left(d_{g}, 1\right)^{T}$

In the case of unloading condition, the unloading plastic flow direction vector $n_{g u}$ is defined as:

$$
\left.n_{g u}=\left(-\mathrm{abs} \frac{d_{g}}{\sqrt{1+d_{g}^{2}}}\right), \frac{1}{\sqrt{1+d_{g}^{2}}}\right)^{T}
$$


$n_{f}=\frac{1}{\sqrt{1+d_{f}^{2}}}\left(d_{f}, 1\right)^{T}$

And $d_{f}$ has a similar expression as

$d_{f}=\left(1+\alpha_{f}\right)\left(M_{f}-\eta\right)$

Where $\alpha_{f}$ are model parameters and $M_{f} / M_{f}$ is equal to relative density.

In fact, it is possible to integrate above directions to obtain both plastic potential and yield surfaces. They are defined as:

$$
\begin{aligned}
& G=q-M_{g} p^{\prime}\left(1+\frac{1}{\alpha_{g}}\right)\left[1-\left(\frac{p^{\prime}}{p_{g}}\right)^{\alpha_{g}}\right] \\
& F\left(\sigma^{\prime}, p_{c}\right)=q-M_{f} p^{\prime}\left(1+\frac{1}{\alpha_{f}}\right)\left[1-\left(\frac{p}{p_{c}}\right)^{\alpha_{f}}\right]
\end{aligned}
$$

Which the size of both surfaces is characterized by the integration constant $p_{g}$ and $p_{c}$.

Plastic modulus is introduced in the following form:

$$
\begin{aligned}
& H_{L}=H_{0} \cdot p^{\prime} \cdot H_{f} \cdot\left(H_{v}+H_{s}\right) \\
& H_{f}=\left(1-\frac{\eta}{\eta_{f}}\right)^{4} \\
& H_{v}=1-\frac{\eta}{M_{g}} \\
& H_{s}=\beta_{0} \beta_{1} \exp \left(-\beta_{0} \xi\right) \\
& \eta_{f}=\left(1+\frac{1}{\alpha_{f}}\right) M_{f}
\end{aligned}
$$

where $H_{L}$ represents the plastic modulus in loading, $H_{0}$ the plastic modulus number, and $H_{f}$, $H_{v}$ and $H_{s}$ the plastic coefficients. $H_{f}$ limits the possible state, $H_{v}$ accounts for the phase transformation, $H_{s}$ considers soil degradation; $\eta_{f}$ is the stress ratio parameter. $\beta_{0}$ and $\beta_{1}$ are material model constants, and $\xi$ is the accumulated plastic deviatoric strain: $\xi=\int\left|d \varepsilon_{s}^{p}\right|$

The reloading plastic modulus $\mathrm{H}$ is given by:

$$
H_{L}=H_{0} \cdot p_{0} \cdot H_{f} \cdot\left(H_{v}+H_{s}\right) \cdot H_{D M}
$$

To consider the history of past loading conditions, plastic modulus has been modified by introducing $H_{D M}$ as:

$$
H_{D M}=\left(\frac{\eta_{r e v}}{\eta}\right)^{\gamma_{D M}}
$$

where $\gamma_{D M}$ is a model constant which has to be calibrated to provide the best prediction of loading-reloading experiments. $\eta_{\text {rev }}$ is the maximum of stress ratio:

- Under unloading condition, the plastic modulus is defined as:

$$
\begin{aligned}
& H_{u}=H_{u 0}\left(\frac{M_{g}}{\eta}\right)^{\gamma_{u}}\left|\frac{M_{g}}{\eta}\right|>1 \\
& H_{u}=H_{u 0}\left|\frac{M_{g}}{\eta}\right| \leq 1
\end{aligned}
$$


where $\gamma_{u}$ is a model constant, $H_{u 0}$ is unloading plastic modulus number, $\eta_{u}$ is the stress ratio from which the unloading takes place.

\section{Symmetrization of Generalized Plasticity Model}

As pointed out, the PZC elasto-plastic model adopted non-associated plastic flow rule which can lead to the asymmetry of the resultant stiffness matrices, because $n \neq n_{g}$ in Eq.(3). It is noticed that if some proportional relationship between $n$ and $n_{g}$ can be established and applied to Eq.(3), the asymmetry of $D^{e p}$ can be removed. In this work, a scalar quantity considering the proportional relationship between $n$ and $n_{g}$ is defined as:

$$
\begin{aligned}
& \left\{r_{1}\right\}=[D]\left\{n_{g L / U}\right\}, \quad\left\{r_{2}\right\}=[D]\{n\} \\
& T=\frac{\left\{r_{1}\right\}^{T} d \varepsilon}{\left\{r_{2}\right\}^{T} d \varepsilon} \beta=H_{L / U}+n^{T}\left\{r_{1}\right\} \\
& D^{e p}=D^{e}-\frac{\left\{r_{1}\right\}\left\{r_{1}\right\}^{T}}{T \beta}
\end{aligned}
$$

In Eq. (26), $D^{e}$ is the symmetric elastic-stiffness matrix, and the numerator of the second term is obviously symmetric. Thus, $D^{e p}$ is a symmetric elasto-plastic stiffness matrix associated with the asymmetric one, in Eq.(3)

\section{Static Analyses}

In order to analysis the earth-rockfill dam static statue in the impounding period, In this study, PZC elasto-plastic model was used to analysis the typical rockfill dam.

The majority of previous research of elasto-plastic numerical models about earth-rockfill dam has been limited to two-dimension cases. Therefore, it is necessary to develop a three-dimensional model to investigate these realistic circumstances. To simulate the dynamic response of three dimensional model, a finite element program, DYNE3WAC[10] (DYNamic Earthquake Analysis Program 3D Window Version for Academic). The program can be used for static, consolidation and dynamic situations under drained and un-drained conditions. DYNE3WAC is an extended version of SWANDYNE II from 2D to 3D, which can effective calculate the geotechnical engineering under static and dynamic condition. DYNE3WAC is the 3D dynamic consolidation finite element program, which is based on the PZC elasto-plastic model, Biot dynamic consolidation theory. Therefore, the three dimensional elasto-plastic program DYNE3WAC can calculate the static and dynamic analysis by the same parameters according to the PZC model.

First, a simplified high earth rockfill dam was discretized and the maximum cross-section of the dam was discretized according to the material zoning and construction design (see Figure 1 ). The three-dimensional dam is conducted by the software, HYPERMESH which can provide the element and node information for the three dimensional finite analysis program DYNE3WAC by the interface which is programed by the FORTRAN.

Figure 2 shows the 3D mesh of the typical dam with 4116 brick and degenerated brick elements and 5054 node, as shown in Fig. 1 and Fig.2. 


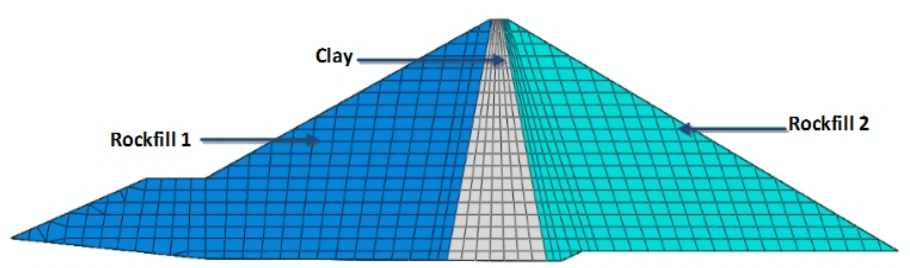

Fig. 1. Material partition

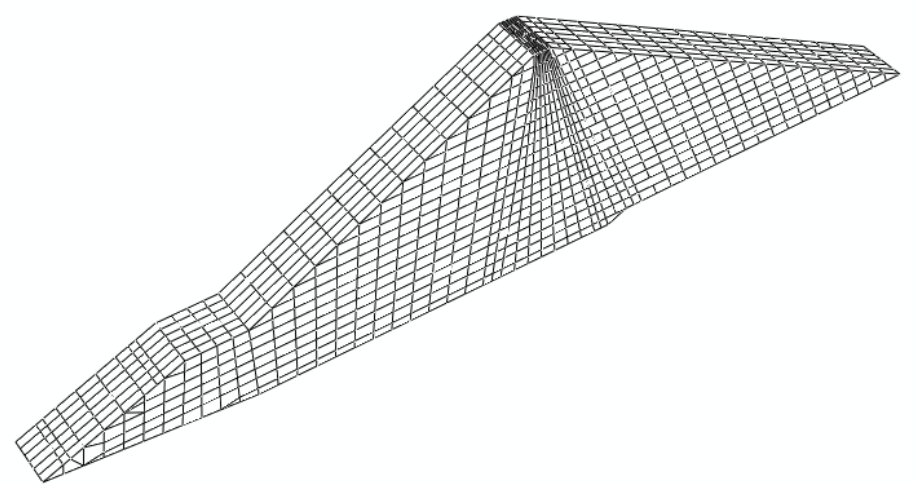

Fig 2. 3D FEM mesh of a Typical dam

Static analysis was conducted on the dam, with programs DYNE3WAC based on PZC model which is widely used in geotechnical and hydraulic engineering. The properties of dam materials are shown in Table 1.

Table 1 Material properties of earth dam soils

\begin{tabular}{lcccccccccccc}
\hline & $K_{o}$ & $G_{o}$ & $M_{f}$ & $M_{g}$ & $\alpha_{g}$ & $\alpha_{f}$ & $H_{0}$ & $\beta_{0}$ & $\beta_{1}$ & $H u_{0}$ & $\gamma_{u}$ & $\gamma_{D M}$ \\
\hline Rockfill 1 & 23.5 & 42.6 & 1.2 & 1.7 & 0.45 & 0.45 & 760 & 4.2 & 0.2 & 0 & 0 & 0 \\
Rockfill 2 & 31.2 & 48.7 & 1.1 & 1.6 & 0.45 & 0.45 & 870 & 4.2 & 0.2 & 0 & 0 & 0 \\
clay & 15.4 & 23.6 & 0.7 & 1.1 & 0.45 & 0.45 & 300 & 0 & 0 & 0 & 0 & 0 \\
\hline
\end{tabular}

The numerical analysis were performed to simulate the performance of the dam during the impounding period with DYNE3WAC finite program. The numerical simulations impounding which upstream water level goes up to the normal storage water level.

Fig. 3 shows the numerical results of finite element analyses. After the reservoir impounded, due to the water pressure on upstream dam, displacement along river directs to the downstream on the whole, and the largest displacement is about $0.8 \mathrm{~m}$. The largest settlement occurs in the middle of core wall with a value of about $2.10 \mathrm{~m}$.

Fig. 4 shows the pore water pressure of the typical dam at the impounding period. 
: (a)

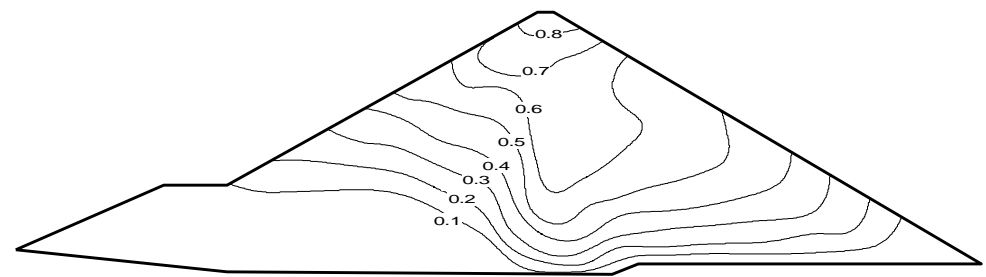

(b)

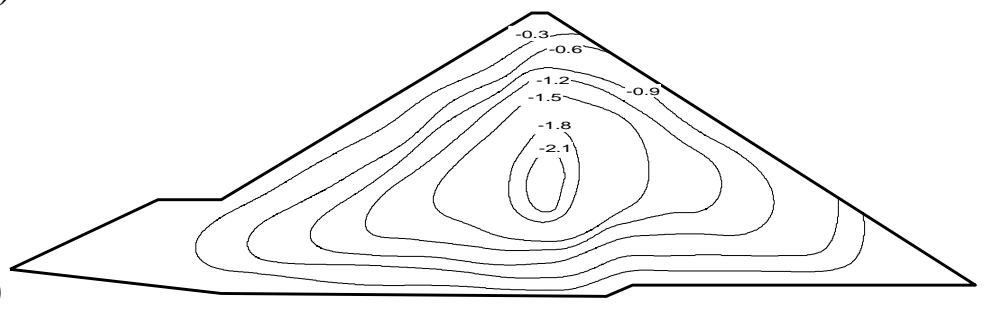

Fig. 3. Displacement distribution of maximum section: (a) displacement along river (m) and (b) vertical displacement $(\mathrm{m})$

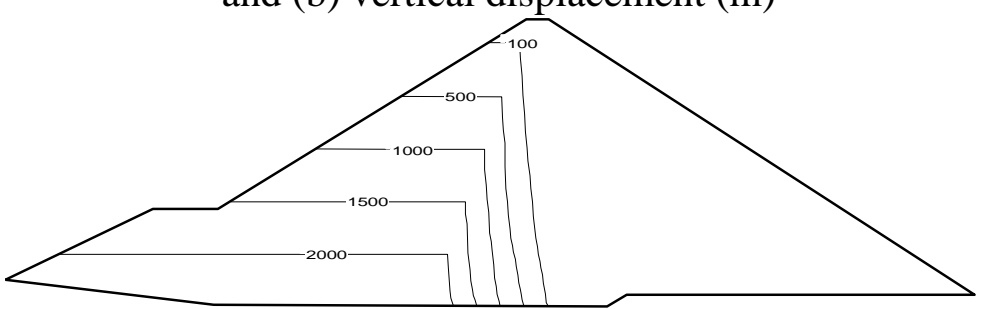

Fig 4. contour lines of pore water pressure $(\mathrm{kPa})$

Because of huge water pressure on upstream dam at the impounding period, the typical dam of horizontal displacement develops toward the downstream and the largest displacement is about $0.8 \mathrm{~m}$. The maximum settlement which is about $2.1 \mathrm{~m}$ occurs in the middle of the typical earth-rockfill dam.

\section{Conclusion}

This paper presents the PZC elasto-plastic constitutive model which can simulate the main material of an typical earth-rockfill dam. Therefore, three-dimensional elasto-plastic finite program DYNE3WAC can analysis the static condition of a typical earth-rockfill dam at the impounding period. The typical dam materials were conducted with PZC model. Static analyses results which included displacement along river, settlement and pore water pressure can show that DYNE3WAC can reasonable calculate three dimensional dam static analysis which can satisfy the earth-rockfill dam displacement and pore water pressure common rules. Dam static analysis results by PZC material which can analysis the dam dynamic condition by the same parameters with the static parameters based on the constitutive model.

\section{Acknowledgements}

The authors would like to gratefully acknowledge Prof. A. H. C. Chan for providing the use of DIANA-SWANDYNE program for the authors. This work was financially supported by the Natural Science Foundation of China (Grant 51379029) and National Major Scientific Research Program in 13th Five-Year Plan(Grant 2016YFB0201001)

\section{REFERENCES}

[1]Li, R.J. A study on the aseismic reinforcement mechanism of stabilizing piles in soil slope. Tsinghua University (2008)

[2] Bian, F.Elasto-plastic FEM analysis of dynamic response of high earth-rockfill dams during earthquake. Tsinghua University (2010) 
[3]Zienkiewicz O C, Mroz Z. Generalized plasticity formulation and applications to geomechanics. Mechanics of engineering materials, 44(3): 655-680 (1984)

[4]Aydingun O, Adalier K. Numerical analysis of seismically induced liquefaction in earth embankment foundations. Part I. Benchmark model[J]. Canadian Geotechnical Journal, 40(4):766-779 (2003)

[5]Aydingun O, Adalier K. Numerical analysis of seismically induced liquefaction in earth embankment foundations. Part II. Application of remedial measures.Canadian Geotechnical Journal, 40(4):766-779 (2003)

[6]Vun, Lee P. Experimental and numerical studies on wave-induced liquefaction to soil around marine structures founded in the seabed. University of Birmingham (2005)

[7] Pastor M, Zienkiewicz O C, Chan A. Generalized plasticity and the modelling of soil behaviour. International Journal for Numerical and Analytical Methods in Geomechanics,14(3): 151-190 (1990) [8]Zienkiewicz O C, Chan A, Pastor M, et al. Computational geomechanics. Wiley Chichester(1999) [9]Frossard E. Uneéquationd'éoulement simple pour les matériauxgranulaires. Géotechnique, 33(1): 21-29(1983)

[10]Zhang X L, Han Y. Numerical Analysis of Seismic Dynamic Response of Saturated Porous Seabed Around a Buried Pipeline[J]. Marine Georesources \& Geotechnology, 31(3): 254-270(2013) 